\title{
A Petri Net approach for representing Orthogonal Variability Models
}

Cristian Martinez, Silvio Gonnet, Horacio Leone

INGAR, Instituto de Desarrollo y Diseño, Santa Fe, Argentina

\{ocmartinez,sgonnet,hleone\}@santafe-conicet.gov.ar

\begin{abstract}
The software product line (SPL) paradigm is used for developing software system products from a set of reusable artifacts, known as platform. The Orthogonal Variability Modeling (OVM) is a technique for representing and managing the variability and composition of those artifacts for deriving products in the SPL. Nevertheless, OVM does not support the formal analysis of the models. For example, the detection of dead artifacts (i.e., artifcats that cannot be included in any product) is an exhaustive activity which implies the verification of relationships between artifacs, artifacts parents, and so on. In this work, we introduce a Petri nets approach for representing and analyzing OVM models. The proposed net is built from elemental topologies that represents OVM concepts and relationships. Finally, we simulate the net and study their properties in order to avoid the product feasibility problems.
\end{abstract}

Keywords: software product line, orthogonal variability model, Petri nets

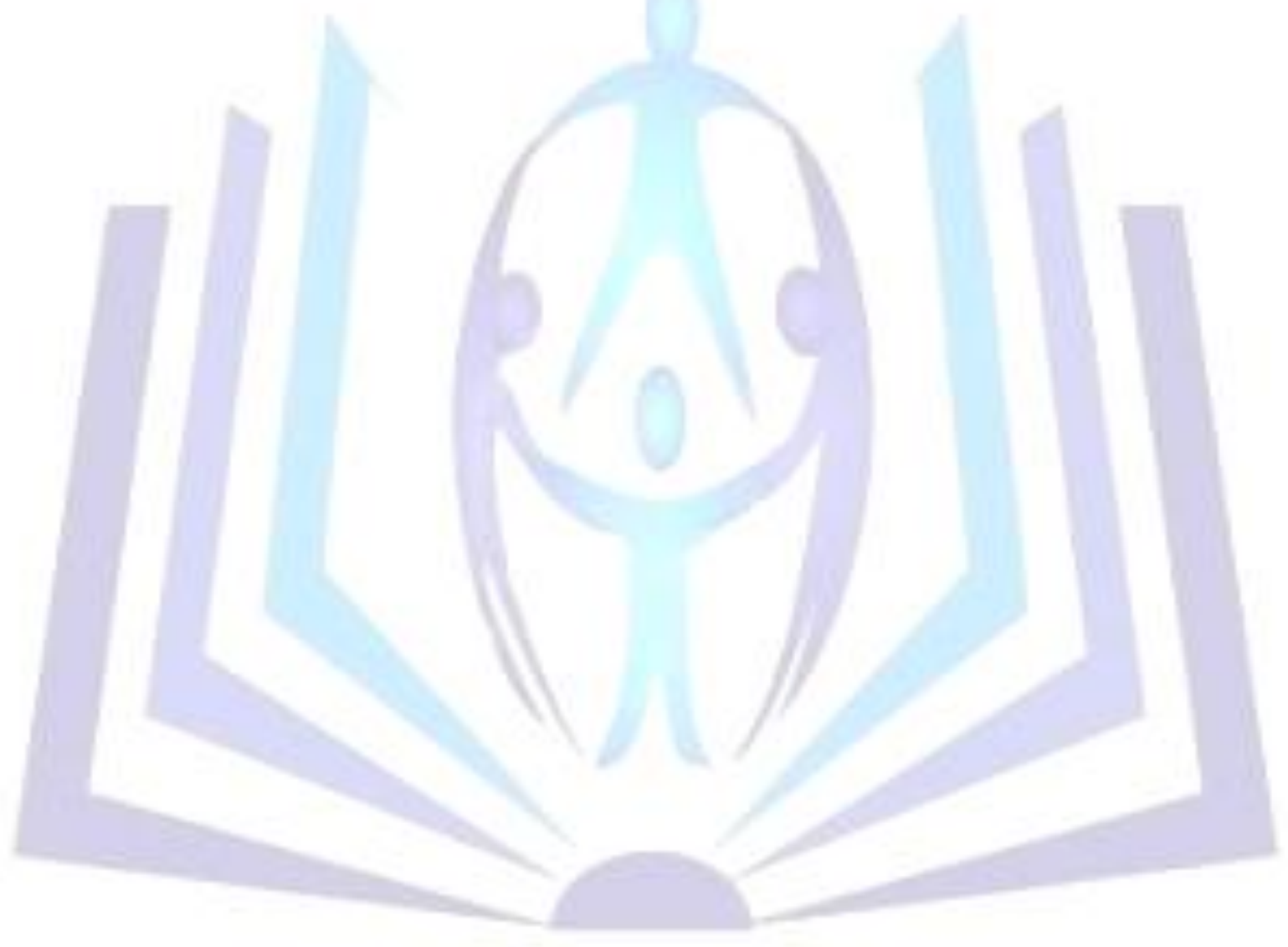

\section{Council for Innovative Research}

\author{
Peer Review Research Publishing System
}

\section{Journal: INTERNATIONAL JOURNAL OF COMPUTERS \& TECHNOLOGY}

\author{
Vol 9, No 1 \\ editor@cirworld.com \\ www.cirworld.com, member.cirworld.com
}




\section{INTRODUCTION}

Software product line engineering (SPLE) has proven to be the methodology for developing a diversity of softwareintensive systems at lower costs, in shorter time, and with higher quality using platforms and mass customization [1]. This is achieved through the management of commonalities and variability in the set of systems' artifacts.

SPLE has two central processes: domain engineering and application engineering.The former is responsible for establishing the reusable platform and thus for defining the variability and the commonality of the product line (PL). The platform consists of all types of software artifacts (requirements, design, realization, tests, etc.). Traceability links between these artifacts facilitate consistent and systematic reuse.The latter process is responsible for deriving product line applications from the platform established in domain engineering. It exploits the variability of the product line and ensures the correct binding of the variability according to the product' specific needs.

Variability can be defined either in a separate variability model or as an integral part of development artifacts. Many contributions have suggested the integration of variability in software development models and diagrams such as class diagrams, feature models and use case diagrams. Nevertheless, it has some disadvantages [2]: the variability spread across different artifacts become almost impossible to keep the information consistent; the single artifacts often leads ambiguous information; an increasing complexity of the software models by adding the variability definitions; a low integration since the concepts used in different kinds of development artifacts differ between them; and the influence of bias in the variability information which come from specific needs of analysis, design, realization, or test artifacts.

Pohl et al. [2] propose a separate model to define the variability of the software product line (SPL). They introduce an Orthogonal Variability Model (OVM) which provides a cross-sectional view of the variability across all software development artifacts. An OVM relates the variability defined to other software development models such as feature models, use case models, design models, component models, and test models.

The idea behind OVM is similar to feature model (FM), but OVM focuses on artifacts relationships whereas FM emphasizes the features decomposition. Therefore we examine some operations (or functions) of automated analysis of feature models (FMs) to be applied in automated analysis of OVM. Kang et al. [3] and Benavides et al. [4] have identified and discussed a complete sets of operations. For this work we only tackle three functions: detection of dead nodes, finding a product, and obtaining all products, however further issues can be covered with our proposal.

(i) Detection of dead nodes: a dead node represents a variation point or variant that never appears in any configuration of a SPL. These unviable nodes lead to inconsistency problems which cause an increase in complexity and a reduction of maintainability.

(ii) Finding a product: this function returns a product which configuration is feasible.

(iii) Obtaining all products: this function returns all possible products. This operation plays a central role during the product line evolution, since all products previously generated must be valid after the changes.

The function (i) refers to a consistency problem while (ii) and (iii) are grouped into satisfiability problem.

Even though these operations will avoid ambiguities and inconsistency, there is still a lack of automated support. Some researches have proposed the use of formal methods to study both FMs $[5,6,7,8]$ and orthogonal variability models $[9$, 10]. Each one of them use different formalism, e.g., propositional logic and constraint programming. These techniques allow the automation check throughout SPLE, both at early stages of development as well as during evolution.

In this work we will introduce a novel approach for automated support of OVM using Petri nets (PNs). The central idea is to represent the dependencies and constraints within in OVM, and next, analyze the properties of the resulting Petri net.

The remainder of this paper is structured as follows. In Section II we present an overview of OVM meta model and Petri net formalism. Section III describes our approach and the proposed topologies. The formalization of the model is given in Section IV. The approach is applied to an example in Section V. Finally, Section VI is dedicated to conclusions and future works, respectively.

\section{OVM META MODEL AND PETRI NETS}

In this section, we introduce the Orthogonal Varibility Model technique and Petri nets formalism.

\subsection{OVM meta model}

An OVM is a model that defines the variability of a software product line. It relates the variability defined to other software development models such as feature models, use case models, design models, component models, and test models [2].

The two central elements of OVM depicted in Fig. 1 are the variation point (VP) and variant (V). A VP documents a variable item (what vary) and a V documents the possible instances of a variable item (how a VP can vary). There are two types of relationships between variation points (VPs) and variants (Vs): variability dependency and constraint dependency.

A variability dependency represents that a VP offers a certain $\mathrm{V}$ and it is specialized into mandatory and optional. The former states that a V must beselected for an application if and only if the associated VP is part of the application. The latter defines that a V can (but does not need to) be a part of the application. A set of Vs that are related through an optional variability dependency (to the same VP) can be grouped into an alternative choice which are constrained by the cardinality maximum and minimum ( $\min$ and max in Fig. 1). 
A constraint dependency documents a restriction that exists between two Vs, a VP and a V, or two VPs; and it is either of the type requires or excludes. An excludes (requires) constraint specifies a mutual exclusion (implication) between two elements.

\subsection{Petri net formalism}

Petri nets are a well-known graphical and mathematical modeling tool [11, 12]. A Petri net (PN) is a directed graph consisting of two kinds of nodes, called transitions and places, where arcs are either from a transition to a place or from a place to a transition.In graphical representation, transition are drawn as boxes and places as circles. Arcs are labeled with their weights (positive integers, $\mathbb{N}$ ), where a $k$-weighted arc can be interpreted as the set of $k$ parallel arcs. Labels for unity weight $(k-1)$ are omitted.A marking $(M)$ assigns to each place a nonnegative integer $\left(\mathbb{N}_{0}\right)$. If a marking assigns to place $p$ a nonnegative integer $k$, we say that $p$ is marked with $k$ tokens.

In this work we use the concept of conditions and events, where places represent conditions, and transitions represent events.A transition (event) has a certain number of input and output places representing the pre-conditions and postconditions of the event, respectively. The presence of a token in a place is interpreted as holding the truth of the condition associated with the place.

\section{THE APPROACH}

In this contribution we introduce a PN approach to represent and study OVMs. The OVM elements and the main activities of application engineering are dealt from an event/condition perspective. To be more precise, the selection of a variation point and the selection of a variant during the product derivation process are represented by the events (transitions), the variation points, variability and constraint dependencies are the pre-conditions (places), and the variants selected are the post-conditions (places).

The goal is to study the behavior of the PN and show the relationships between their markings $(\boldsymbol{M})$ and the valid configurations of the underlying OVM. The interesting $\boldsymbol{M}$ are those which no transitions are enabled (leaf nodes in the reachability graph), in other words, all decisions about the inclusion of variation points and variants have been taken. Although the following PNs belong to trivial OVMs, they can also be combined to support models with increasing complexity.

We briefly introduce the notation used throughout the paper. Given the place $\boldsymbol{p}_{\mathbf{1}}$ represents the variant $\boldsymbol{V}_{\mathbf{1}}, \boldsymbol{M}$ the marking, and $\boldsymbol{M}\left(\boldsymbol{p}_{1}\right)$ the number of tokens in $\boldsymbol{p}_{1}$, whereas $\boldsymbol{M}\left(\boldsymbol{p}_{1}\right)=\mathbf{1}$ depicts the consideration of the variant $\boldsymbol{V}_{\mathbf{1}}, \boldsymbol{M}\left(\boldsymbol{p}_{1}\right)=\mathbf{0}$ indicates the no inclusion of $\boldsymbol{V}_{\mathbf{1}}$. The firing sequence $\boldsymbol{\sigma}$ is the chain of events (selections) to reach that marking.

\subsection{Variability dependency}

A variability dependency is an association between a variation point and a variant, and it is specialized into mandatory and optional (Fig. 1).

\subsubsection{Mandatory}

A mandatory dependency states that the consideration of a VP implies the inclusion of the Vs associated to that VP. 
In the OVM shown in Fig. 2 (a), the variants $V_{1}, V_{2}, \ldots V_{n}$ are associated to $V P_{1}$ through mandatory dependencies. There are two configurations $\left\{\varnothing,\left(V_{1}, V_{2}, \ldots V_{n}\right)\right\}$. The former $(\varnothing)$ does not include the variation point. The latter configuration $\left(V_{1}, V_{2}, \ldots V_{n}\right)$ considers $V P_{1}$ together with all variants.

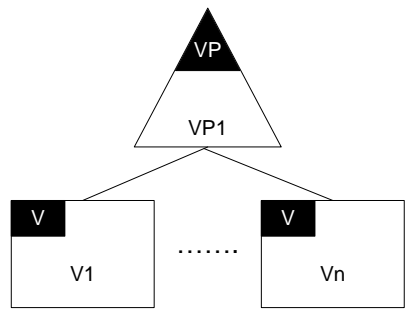

(a)

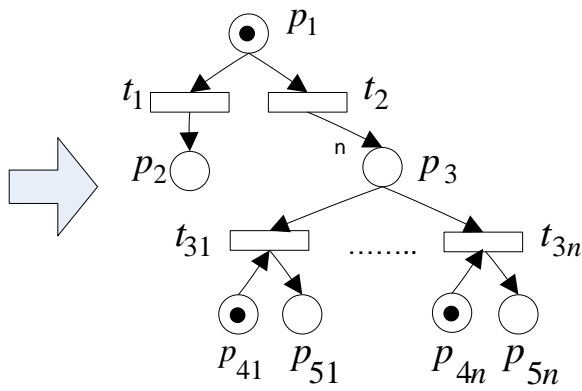

(b)

Fig 2: Mandatory dependency. In (a) $V P_{1}$ and their mandatory variants $V_{1}, V_{2}, \ldots V_{n}$. In (b) the topology proposed.

In the PN illustrated in Fig. 2 (b) the place $p_{1}$ represents $V P_{1}$, the transitions $t_{1}$ and $t_{2}$ the events no-selection and selection of $V P_{1}$ respectively. Each $t_{3 i}$ corresponds to the selection of the variant $i$. Places $p_{4 i}$ constraint the maximum number of selection of a variant (OVM allows up to 1 instance for each variant), and places $p_{5 i}$ indicates the selection of the variant $i$. Finally, $p_{3}$ enables the variant selection transitions.

The only one token in $p_{1}$ enablest $t_{1}$ and $t_{2}$, but exactly one can be fired. If $t_{1}$ fires, no other transitions is enabled and $V P_{1}$ is not included. The marking $M\left(p_{51}\right)=M\left(p_{52}\right)=M\left(p_{5 n}\right)=0$ corresponds to the configuration $\emptyset$.Otherwhise, if $t_{2}$ fires, $n$ tokens are put in $p_{3}$ and transitions $t_{31}, \ldots t_{3 n}$ are enabled.After firing the transitions, $M\left(p_{51}\right)=M\left(p_{52}\right)=M\left(p_{5 n}\right)=1$ which represent the configuration $\left(V_{1}, V_{2}, \ldots V_{n}\right)$.

\subsubsection{0ptional}

In an optional dependency the consideration of a VP does not imply necessarily the inclusion of the V.

In the OVM shown in Fig. 3 (a), the variation point $V P_{1}$ is related through an optional variability dependency to the variants $V_{1}, V_{2}, \ldots V_{n}$. The possible configurations are: $\emptyset$ and $V P_{1} \cup \mathcal{P}\left(\left\{V_{1}, V_{2}, \ldots V_{n}\right\}\right)(\mathcal{P}$ indicates the power set).

The topology (Fig. 3 (b)) is similar to the above PN (Fig. 2 (b)) but is extended with the addition of $t_{4}, p_{6}$, and $p_{7}$. $t_{4}$ corresponds to the event no-selection of variant, and $p_{6}$ and $p_{7}$ indicate the maximum event occurrences and the effective occurrences respectively. In this case the number of tokens of $p_{6}$ is the amount of variants $(n$ in Fig. 3 (b)).

As stated above after firing $t_{1}$ no other transitions is enabled. The resulting marking is $M\left(p_{51}\right)=M\left(p_{52}\right)=M\left(p_{5 n}\right)=0$ and no variants is included (configuration $\varnothing$ ). Otherwise, if $t_{2}$ is fired,ntokens are put in $p_{3}$, and transitions $t_{31}, \ldots t_{3 n}$ are enabled. After selecting the variants, the rest of tokens in $p_{3}$ will be consumed by $t_{4}$.

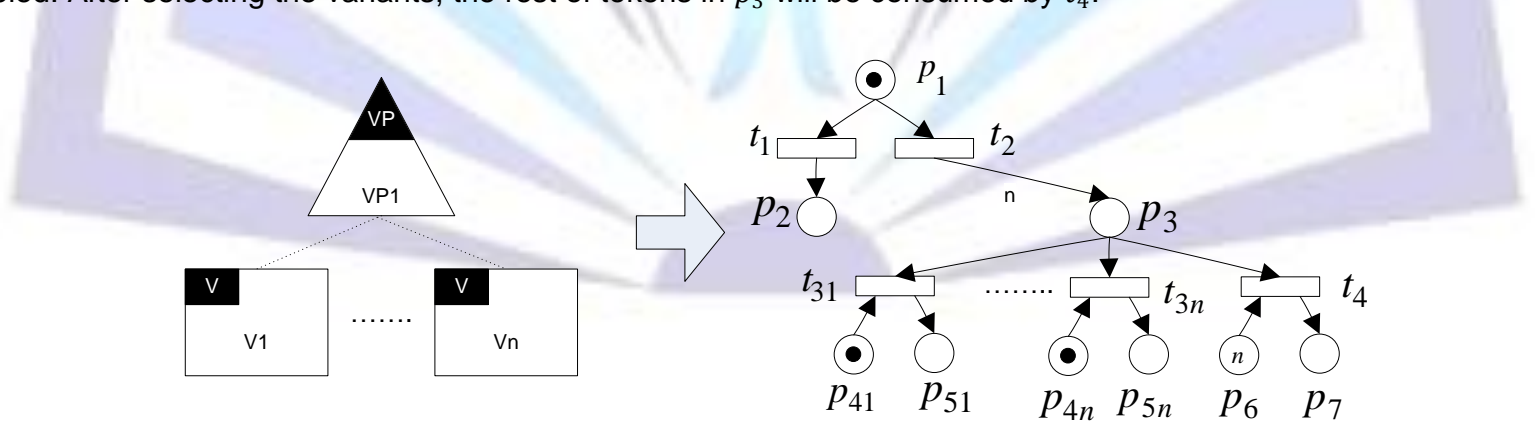

(a)

(b)

Fig 3: Optional dependency. In (a) the $V P_{1}$ and their optional variants $V_{1}, V_{2}, \ldots V_{n}$. In (b) the topology proposed.

\subsubsection{Alternative choice}

An alternative choice groups a set of $\mathrm{Vs}$ and defines a range $[m . . n]$ for the amount of $\mathrm{Vs}$ to be selected, with $m$ and $n$ the minimum and maximum cardinality respectively ( $\min$ and $\max$ in Fig. 1).

The topology is similar to the previous PN (Fig. 3 (b)) but the initial marking of $p_{6}$ is $n-m$. It means that at least $m$ variants must be included. An alternative choice will be show in the case study in Section 5.

\subsection{Constraint dependency}


A constraint dependency documents a restriction that exists between two Vs, between a VP and a V, or between two VPs. Each restriction is either of the type requires or excludes.
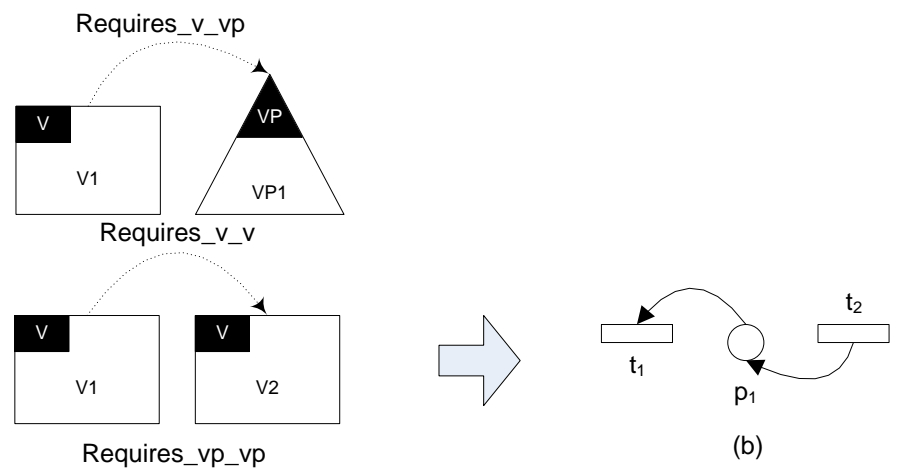

(b)

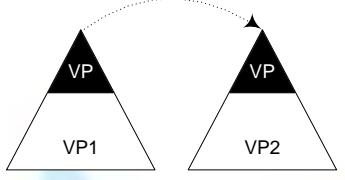

(a)

Fig 4: Requires dependency. In (a) the three types of requires constraints and their topology (b).

\subsubsection{Requires constraint}

In a requires constraint the consideration of a variation point (or variant) implies the inclusion of another variation point (or variant).

Fig. 4 (a) illustrates the dependencies Requires_v_vp, Requires_v_v and Requires_vp_vp and (b) thetopology proposed. The transitions $t_{1}$ and $t_{2}$ represents the events selection of variation point (or variant) and the place $p_{1}$ corresponds to the constraint. After firing $t_{1}$ (event unrestricted), $t_{2}$ (event restricted) is enabled.

\subsubsection{Excludes constraint}

A excludes constraint indicates that two variation points, two variants or a variation point and a variant are mutually exclusive.

Fig. 5 (a) illustrates the dependencies Excludes_v_vp, Excludes_v_v and Excludes_vp_vp and (b) the topology proposed. The transitions $t_{1}$ and $t_{2}$ represents the events selection of variation point (or variant) and the place $p_{1}$ indicates the constraint. The token in $p_{1}$ enables $t_{1}$ and $t_{2}$ but only one can be fired.
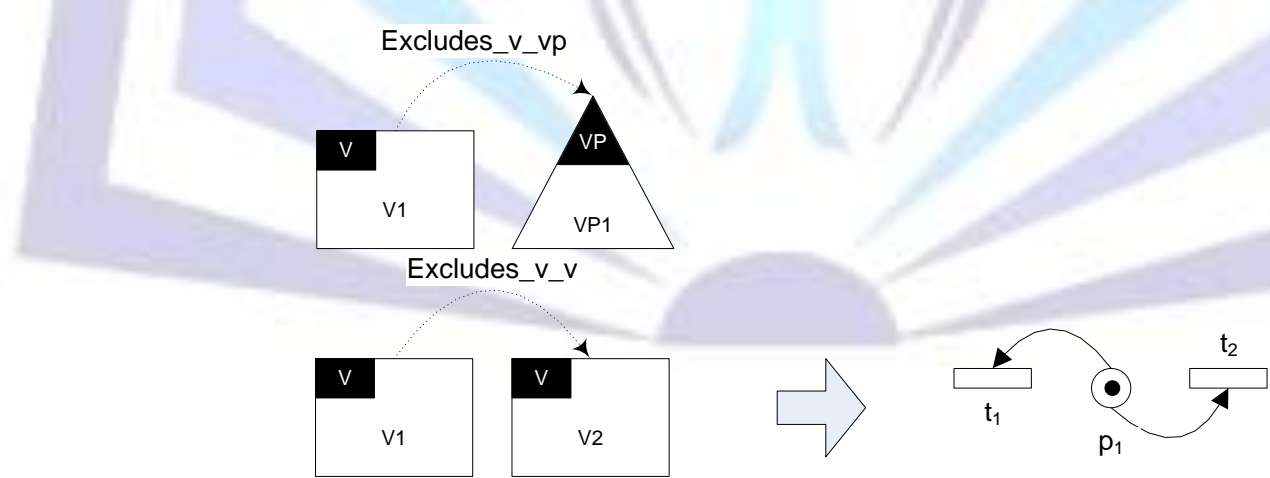

Excludes_vp_vp

(b)

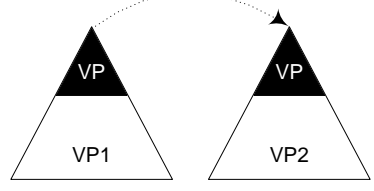

(a)

Fig 5: Excludes dependency. In (a) the three types of excludes constraints and their topology (b).

\section{CONCEPTS AND PROPERTIES OF THE TOPOLOGY PROPOSED}

In this section we provide PNs concepts and properties in terms of OVM. 


\subsection{Concepts}

The meaning of some Petri net concepts helps to understand the relationship between the dynamic of the PN and the configurations allowed by the OVM.

\subsubsection{Marking}

A marking is am-vector where $m$ is the total number of places. The $i$-th component of $M(M(i))$, is the number of tokens in place $i$. Each $M$ depicts a specific product (or configuration) of the product line and tokens indicate which variation points and variants are included.

The interesting markings are those with no transition enabled denoted by dead-end. In these markings all decisions have been made and there are not unresolved variabilities.

\subsubsection{Token}

The presence of a token in a place has several meanings according to the OVM concept associated. In the case of variation point (variant) a token states that the variation point (variant) is included. In a constraint dependency, the token ensures the accomplishment of mutually exclusive (inclusive) constraint disabling (enabling) a transition after firing another one. In the case of cardinality, the number of tokens in a place restrict the maximum or minimum of transition that can be fired, when the place is empty no more variants can be selected.

\subsubsection{Firing sequence}

The firing sequence from the initial marking to a dead-end points out the sequence of transition (or events) to reach a marking. This sequence gives information about the selection of VP and selection of V necessary to set a configuration. Of course, not all sequences are possible since dependencies gives rules, e.g., the selection of a variation point is previous to the selection of their variants.

\subsection{Properties}

One of the major strength of Petri nets is their support for analysis of problems and properties associated with dynamic systems. We focus on those properties which are closely related with the functions identified in Section I. In the following we will explain how boundedness, reachability and liveness allow to deal with consistency and satisfability problems.

\subsubsection{Liveness}

This property is related with the absence of deadlocks. There are five different levels of liveness, we focus on $L 1$-live also called potencially firable. A PN is said to be L1-live if all transitions can be fired at least once in some firing sequence.

In the topologies proposed each variation point and variant is associated to some transition; therefore if exist at least one firing sequence for any VP and V, there is not any dead node. L1-live provides sound basis for function (i) detection of dead nodes.

\subsubsection{Reachability}

A reachability graph $(R G)$ of a Petri nets contains all possible reachable markings for a given initial marking. Nodes represent markings and its successors and each arc indicates the transitions which transforms one marking to another. This property helps to find all possible configurations of a product line and allows to deal with functions (ii) finding a product and (iii) obtaining all products.

\subsubsection{Boundedness}

The reachability is closed related with the boundedness. A reachability graph of a PN has a finite number of states if and only if the PN is bounded. A net with this property allows to discover all possible products and no overflow occurs.

\section{CASE STUDY: ELECTRONIC PAYMENT}

The following case study describes partially the variability of aelectronic payment (e-payment) for a software product line. Initially we introduce the OVM diagram which takes only the payment aspects, then build a Petri net for representing the OVM diagram and we finish studying their properties.

\subsection{OVM diagram for electronic payment}

The OVM diagram illustrated in Fig. 6 presents the variation points: $e$-payment $\left(V P_{1}\right)$, cash machine $\left(V P_{2}\right)$ and $\operatorname{security}\left(V P_{3}\right)$. The variant debit card $\left(V_{1}\right)$ is mandatory for $V P_{1}$. The variants $h t t p s\left(V_{2}\right)$ and $\operatorname{ssl}\left(V_{3}\right)$ are optional for $V P_{3}$ and defines an alternative choice with range [1..1]. The variants associated to $V P_{2}$ does not affect the rest of dependencies so they were not included in the OVM diagram. Finally the constraint dependencies shown the mutually exclusion between $V P_{2}$ and $V_{2}\left(\right.$ Exludes_vp_vp) and the implication between $V P_{1}$ and $V P_{3}($ Requires_vp_vp). 
Requires_vp_vp
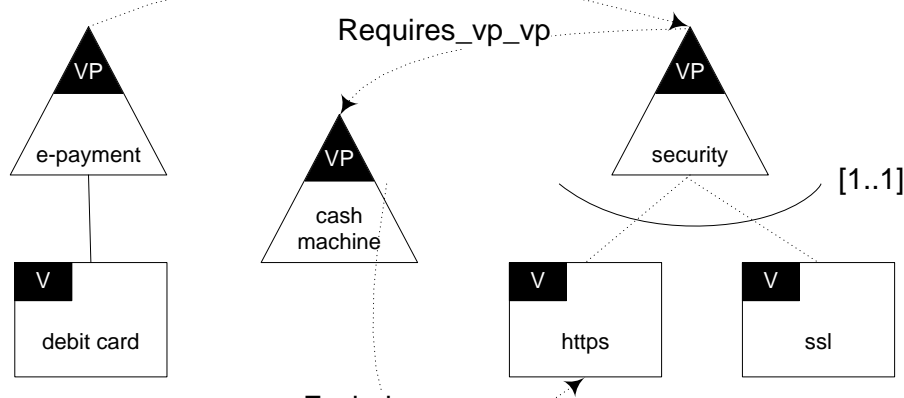

Excludes_vp_v

Fig 6: Case study: electronic payment

\section{2 $P N_{O V M}$ for electronic payment}

The PN for electronic payment is shown in Fig. 7. The places $p_{1}, p_{6}$ and $p_{8}$ corresponds to the variation points $V P_{1}, V P_{2}$ and $V P_{3} ; p_{5}, p_{12}$ and $p_{14}$ represents the variants $V_{1}, V_{2}$, and $V_{3} ; p_{15}$ is the constraint dependency Excludes_vp_v $\left(V P_{2}-V_{2}\right)$; and $p_{17}, p_{16}$ corresponds to the constraint dependencies Requires_vp_vp $\left(V P_{1}-V P_{3}\right.$ and $\left.V P_{3}-V P_{2}\right)$.

The interpretation of the places $p_{2}, p_{7}, p_{9}, p_{3}, p_{10}, p_{4}, p_{11}$ and $p_{13}$ is not directly observable from the OVM diagram and their meaning is related to the cardinalities and rules explained in Section III.

The transitions $t_{2}, t_{5}$ and $t_{6}\left(t_{1}, t_{4}\right.$ and $\left.t_{7}\right)$ represents the selection (no-selection) of variation point; and $t_{3}, t_{8}$ and $t_{9}$ corresponds to the selection of variant $V_{1}, V_{2}$ and $V_{3}$ respectively.

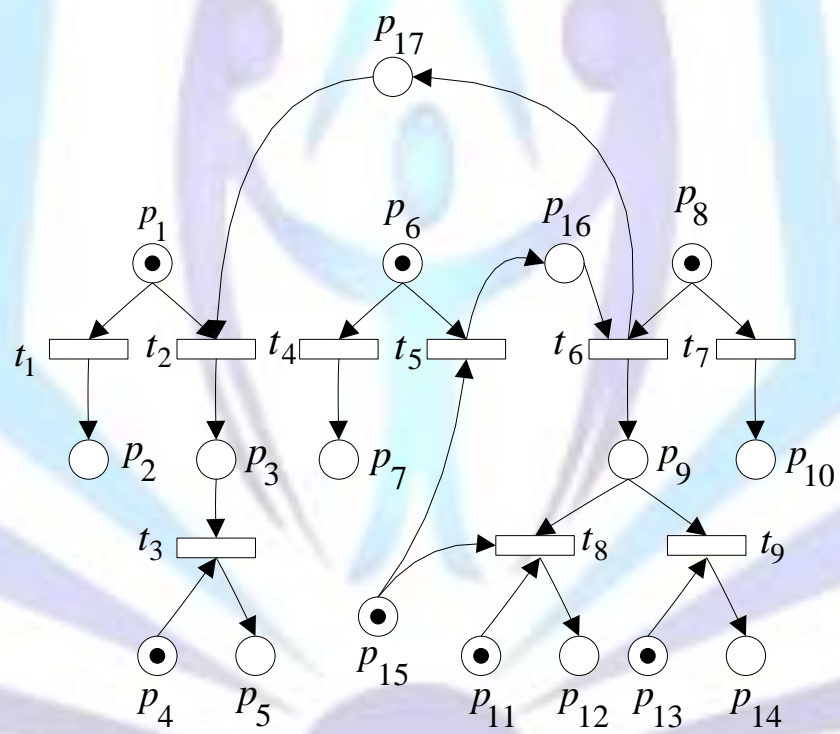

Fig 7: OVM for electronic payment 


\subsection{Analysis of $P N_{O V M}$ electronic payment using the reachability graph}

The Fig. 8 (a) illustrated the reachability graph of the $P N_{O V M}$ electronic payment. For simplicity the nodes only described a subset of places, those which corresponds to variation points, variants and constraint dependencies.

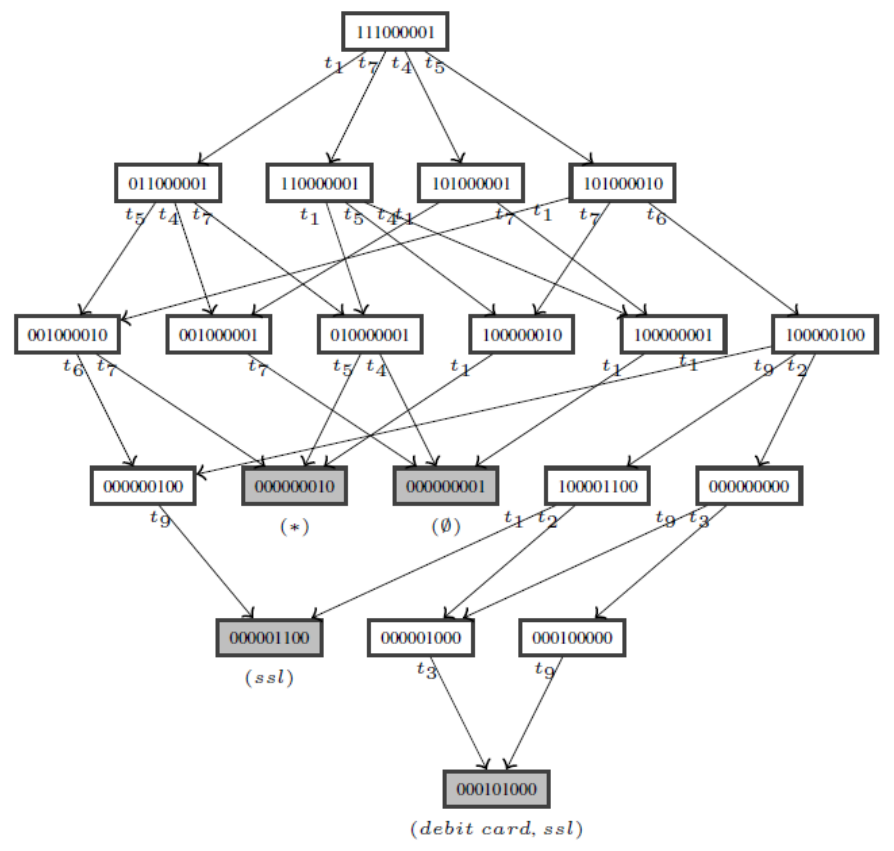

(a)

\begin{tabular}{|c|c|}
\hline 000000001 & $\sigma=t_{7} t_{4} t_{1} \rightarrow(\emptyset)$ \\
\hline 000001100 & $\sigma=t_{5} t_{6} t_{9} t_{1} \rightarrow(s s l)$ \\
\hline
\end{tabular}

(b)

Fig 8:PN $N_{\text {OVM }}$ of e - payment. (a) shows the reachability graph, and (b) dead-ends and firing sequence.

The top node (111000001) is the initial marking and the terminal nodes (gray scale) are the four affordable configurations in the software product line, for example 000101000 indicates the configuration \{debit card, ss/\}. The reachability graph also provides information regarding the sequence of events to reach such configurations. The independence of certain events (those without any constraint dependency) results in several paths to the same dead-end. Fig. 8 (b) shows some firing sequence and the corresponding dead-end node.

By examining the graph we can get information about the consistency and satisfability of the underlying OVM. All transitions concerning variation points $\left(t_{2}, t_{5}\right.$ and $\left.t_{6}\right)$ can be fired in some sequence, then all variation points live. However the situation is certainly different in the case of variants, whereas the transitions $t_{3}$ and $t_{9}$ are firable, $t_{8}$ is nonfirable for any firing sequence, thus $h t t p s$ will never be included during the product derivation.With respect to satisfability, the set of configuration allowed is $\left\{\varnothing,(s s l)\right.$, (debit card, ssl)\} (shown in Fig. 8 (b)). Note that configuration $000000010\left(^{*}\right)$ is related to $V P_{2}$ which is not completely covered in this example.

\section{CONCLUSIONS AND FUTURE WORK}

Some general problems and operations of feature modeling identified in [3] and [4] can be observed in OVMs as well. In that sense, this paper introduced a Petri net approach for representing and studying OVMs during the development and evolution of a software product line. We defined set of elemental topologies of PNs which dealts the OVM concepts and rules from a event/condition perspective. Then, we focused on Petri net properties and shown that liveness, reachability and boundedness provide a sound basis for analyzing satisfiability and consistency functions mentioned above. Finally, the case study electronic-payment was developed using our approach and reported that the variant debit card will never be included in any product.

An important challenge is the size of the variability models. Benavides et al. [4] observe an ascendant tendency in the amount of features in last years, from 15 features used in 2004 up to 300 features in 2010 . The increasing complexity plays a key role in the evaluation of techniques and tools. Future work will be address toward the use of reduction rules for Petri nets in order to facilitate the analysis by reducing the system model to a simpler one, while preserving the properties.

Another trend is to study other Petri net properties such reversibility and synchronic distance. The reversibility could help us to recover the initial marking given the possible configurations (or products). Synchronic distance is a metric closely related to a degree of mutual dependence between two events, and could provide qualitative information of product line variability.

A third issue is to extend the topologies proposed to feature modeling. Since FMs and OVMs share variability and dependency constraints (e.g., excludes, includes, and alternative choice) we will applied the same event/condition perspective of Petri nets for studying feature models. 


\section{ACKNOWLEDGEMENTS}

The authors wish to acknowledge the financial support received from CONICET,Universidad Tecnológica Nacional and Agencia Nacional de Promoción Científicay Tecnológica (PAE-PICT 02315).

\section{REFERENCES}

[1] Northrop, L., Clements, P. 2009. A framework for Product Line Practive. Version 5.0. Software Engineering Institute. Carnegie Mellon University. http://www.sei.cmu.edu/productlines/frame report/index.html (ver. 06/24/2013).

[2] Pohl, K., Böckle, G., van der Linden F. 2005. Software Product Line Engineering: Foundations, Principles, and Techniques. Springer Heidelberg.

[3] Kang, K., Cohen, S., Hess, J., Novak, W., Peterson S. 1990. Feature-Oriented Domain Analysis (FODA) Feasibility Study. Technical Report CMU/SEI-90-TR-21. Software Engineering Institute. Carnegie Mellon University.

[4] Benavides, D., Segura, S., Ruiz-Cortés A. 2010. Automated analysis of feature models 20 years later: A literature review. Journal of Information Systems 35 (2010) 615-636.

[5] Batory, D. 2005. Feature models, grammars, and propositional formulas. In Software Product Lines Conference.

[6] Sun, J., Zhang, H., Li, Y., Wang H. 2005. Formal semantics and verification for feature modeling. In Proceedings of ICECSS05.

[7] Benavides, D., Ruiz-Cortés, A., Smith, B., O'Sullivan, B., Trinidad, P. 2006. Computational issues on the automated analyses of feature models using constraint programming. International Journal of Software Engineering andKnowledge Engineering.

[8] Benavides, D., Ruiz-Cortés, A., Trinidad, P. 2005. Using constraint programming to reason on feature models. In the $17^{\text {th }}$ International Conference on Software Engineering and Knowledge Engineering.

[9] Metzger, A., Heymans, P., Pohl, K., Schobbens, P., Saval, G. 2007. Disambiguating the Documentation of Variabilityin Software Product Lines. In the $15^{\text {th }}$ IEEE International Requirements Engineering Conference, pp. 243253.

[10] Lauenroth, K., Pohl, K. 2008. Dynamic Consistency Checking of Domain Requirements in Product Line Engineering. In the $16^{\text {th }}$ IEEE International Requirements Engineering Conference, pp. 193-202.

[11] Murata, T. 1989. Petri Nets: Properties, Analysis and Applications. Proceedings of the IEEE, Vol. 77:4.

[12] Peterson, J. 1977. Petri Nets. ACM Computing Surveys, Vol. 9:3. 